\title{
NOVEL THREE-DIMENSIONAL SURFACE MICROFLUIDICS ENABLED BY UNCONVENTIONAL FLUIDIC INTERFACES
}

\author{
Lingfei Hong ${ }^{1,2}$, and Tingrui Pan ${ }^{1 *}$ \\ ${ }^{1}$ Department of Biomedical Engineering, University of California, Davis, California, USA \\ ${ }^{2}$ School of Instrumentation Science and Optoelectronics Engineering, Beihang University, Beijing, China
}

\begin{abstract}
Surface microfluidics, offering unique flow characteristics and addressing intrinsic interfacial problems encountered in microfluidic systems, becomes an attractive alternative to the conventional closed-channel counterparts. Moreover, the planar structure in surface microfluidics enables simple microfabrication, while the open-surface platform allows direct fluidic operations and easy surface modification. In the paper, we first present novel three-dimensional microfluidic manipulations utilizing the unique flexible gas-liquid interface on surface microfluidic platform. The surface-micropatterned triple lines, defining the gas/liquid/solid interfacial boundaries, play an important role in determining the microflow profile. Specifically, the distinct elastic gas-liquid interface enables time-dependent (capacitive) and threedimensional (deformable) operations of the flow, in contrast to the primary linear (resistive) nature of conventional microfluidics. Spatiotemporally dependent flow patterns on the planar microfluidic surfaces have been theoretically analyzed and experimentally controlled. Utilizing the distinct flow characteristics, new surface microfluidic functionalities, such as microflow regulation and flow rate-controlled switching, have been established. Furthermore, 3D surface microfluidic networks have been fabricated by fluidic connection of two independent planar surfaces, and microflow multiplexers with two-level flowswitching have been successfully devised to illustrate its potential in digital microfluidics.
\end{abstract}

\section{INTRODUCTION}

Microfluidics has been widely employed in chemical and biological analyses over the past decades [1-2]. Constructed by a wide variety of microfabrication techniques, microfluidic devices serves an attractive alternative to conventional fluidic systems and offers desirable features, including miniature amount of reagents, accelerated reaction speed and efficiency, and integratableprocessing capability. Basic fluidic functions, e.g., mixing, pumping, and switching, have all been implemented by a number of active and passive fluidic designs [3-7]. However, a large majority of microfluidic devices, which utilizes the closed-channel configuration and requires spatial confinement of flow, usually involves complex multilayer microfabrication and packaging of the fluidic conduits. Moreover, any gas-liquid interface (e.g., bubble trapping) in microscale channel could present unexpected hurdles to microfluidic operations, which becomes further problematic with dimension down-scaling.

Surface microfluidics, an effective alternative to addressing the aforementioned issues encountered in conventional microfluidics, has received increasing attention from microfluidic community recently [8-10]. Enabled by the latest advances in interfacial sciences and surface chemistry, planar microfluidic pathways are defined lithographically by the contrast of interfacial energies instead [11-14]. The boundaries among solid/liquid /gas phases, known as triple line, serve as virtual borders of fluid flow. Majority of surface microfluidic devices, also known as surface directed channels, are established by confining flow between two surfaces of identical micropatterns with wettability contrast to the substrates [11-12, 14-15]. The similar concept is first demonstrated in a two-immiscible microfluidic system in a closed channel configuration [15]. Further investigations focus on different micropatterning techniques of wettability contrast. A variety of micromachining techniques have been successfully demonstrated to define hydrophilic patterns on hydrophobic substrates or hydrophobic patterns on hydrophilic substrates, including direct writing by retrofitted pen $[14,16]$, photolithography [11, 17], lift-off [4, 12], inkjet printing [18-19], microplasma writing [13], and deposition [20]. In comparison with the existing surface patterning methods, our group has previously developed a one-step photopatterning technique to achieve ultrahigh contrast wettability on a wide variety of surfaces. Using a photosensitive superhydrophobic nanocomposite formula (with water contact angle $>165^{\circ}$ ), surface microfluidics has been established on single planar substrate using the superhydrophobic micropatterns, which comes with one unrestricted elastic gas-liquid interface [9, 21]. The complete open-surface platform permits direct environmental access, flexible fluidic operations and simple surface modification, while the single planar structure of surface microfluidics enables one-step microfabrication without packaging or alignment.

Considering the substantial fluidic differences between surface and closed-channel flows, more in depth microfluidic analysis is of essential importance to the emerging direction. Furthermore, the distinct elastic gas-liquid boundary could potentially enable novel spatiotemporal control of the fluid due to the presence of fluidic compliance (capacitance) and pressuredependent resistance, which is usually negligible in conventional system. In this paper, we present analytical and experimental investigation of surface microfluidics with emphasis on the spatiotemporal manipulation of the elastic gas-liquid interface. The planar fluidic circuits are defined by the interfacial triple lines of microfabricated superhydrophobic structures on hydrophilic substrate. Moreover, the elastic gas-liquid boundary adds vertical dimension and thereby lateral compliance to the laminar microflow, analogous to stray capacitance in electronic circuits, and therefore, the pressure gradient not only propels the fluid but controls the cross-sectional profile of the flow. In addition, the compliance of the interface introduces temporal dependence of the fluidic field. Based on the unique three-dimensional and timedependent flow characteristics, new microfluidic operations have been achieved through the interfacial manipulation, including microflow regulation and flow rate-controlled switching. Furthermore, 3D surface microfluidic networks have been fabricated by fluidic connection of two independent planar surfaces, and microfluidic multiplexers with three-level flowswitching have been successfully devised to illustrate its potential in digital microfluidics.

Figure 1 shows a prototype of a surface microfluidic device with parallel 3D spiral flows. As can be seen, it consists of two micropatterned superhydrophobic substrates placed face-to-face with a spacer. Colored fluids are directed by the surface guided microchannels on one side and connected through miniature vertical capillary bridge towards the other side. Running up-anddown between the top and bottom surfaces, the streams develop into 3D spiral flow patterns without intercepting with each other. 


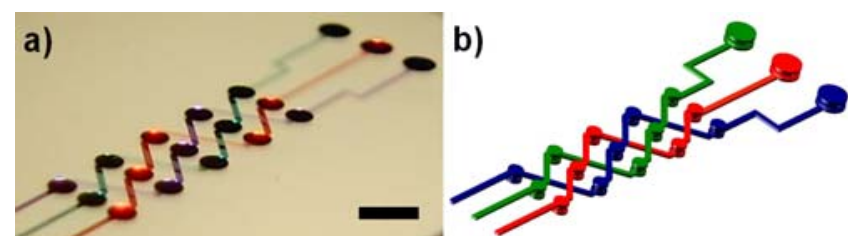

Figure 1: (a) Prototype of a surface microfluidic device with parallel spiral flows along with (b) schematic illustration of unintercepted $3 D$ flow streams. (Scale bar: $2 \mathrm{~mm}$ )

\section{THEORETICAL ANALYSIS}

As aforementioned, elastic boundary of the gas-liquid interface in surface microfluidics introduces lateral fluidic compliance/capacitance as well as pressure-dependent resistance, unlike its closed-channel counterpart, and therefore, the linear flow-pressure relationship (i.e., Poiseuille's equation) is no longer valid. To analyze surface microfluidic networks, a lumpedelement equivalent model has been developed. Similar to the transmission line theory, the distributed-parameter network is used to describe the resistive and capacitive nature of the fluidics in an infinitesimal length. More complicatedly, resistance $(\boldsymbol{R})$ and capacitance $(\boldsymbol{C})$ of the surface microfluidic network is flowdependent or pressure-dependent (i.e., resistance and capacitance vary with flow rate or pressure). For example, in a straight microchannel, flow through the resistive solid-liquid boundary generates pressure gradient. At the meanwhile, deformed by the induced pressure change, the elastic gas-liquid interface accommodates fluidic accumulation and alters the cross-sectional area. As a consequence, the flow resistance is adaptively modified by the influx. Figure 2(a) represents a general lumped-element model of a fluidic component using distributed parameters.

a)

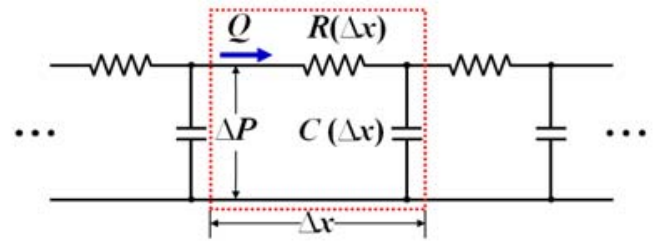

b)

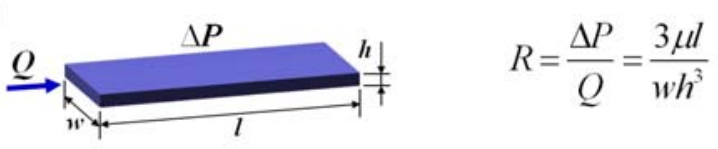

c)

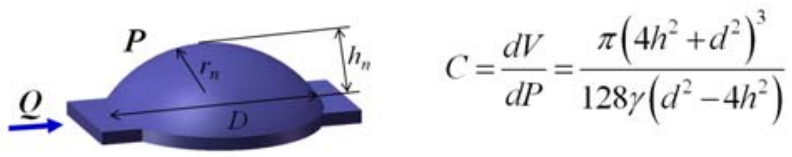

Figure 2: (a) A general lumped-element model describing a surface microfluidic component with both distributed resistive and capacitive elements, (b) the resistive model of a straight channel with unaltered boundary, (c) the capacitive model of a circular reservoir with deformable interface.

More importantly, despite the complex nature of surface flow, the pressure/flow dependence can be applied to tailor the resistive and capacitive characteristics of individual fluidic components. In general, at low flow rate (with small pressure drop), straight microchannels can be approximated as primarily resistive elements as long as the deformation of the gas-liquid interface is insignificant. Thus, the fluidic resistance of a microchannel with one unaltered but frictionless boundary can be calculated according to the classic laminar flow theory $R=\frac{3 \mu l}{w h^{3}}$, where $\mu$ is the dynamic viscosity of the fluid, $\boldsymbol{l}, \boldsymbol{w}$, and $\boldsymbol{h}$ are the length, width and height of the microchannel, respectively. In contrast, the profile of a circular reservoir with relative high surface-to-volume ratio is subject to even a slight pressure change, which deforms the gasliquid interface into a spherical dome with the radius of curvature predicted by Laplace equation $\mathbf{1} \boldsymbol{r}=\Delta \mathbf{P} / \mathbf{2} \boldsymbol{\gamma}(\boldsymbol{\gamma}$ is the fluidic surface tension). As a result, under small surface flow, the reservoir behaviors major capacitive characteristics, which can be expressed as

$$
C=\frac{d V}{d P}=\frac{\pi\left(4 h^{2}+d^{2}\right)^{3}}{128 \gamma\left(d^{2}-4 h^{2}\right)}
$$

where $\boldsymbol{h}$ is the height of accumulated liquid, and $\boldsymbol{d}$ is the diameter of the reservoir. Figure 2 (b) and (c) show the geometrical design parameters used to determine the resistive (microchannel) and capacitive (reservoir) flow elements, respectively.

Incorporating the distinct capacitive and resistive characteristics into surface microfluidic design, novel fluidic operations (e.g., first-order or higher-order pressure-flow responses) can be implemented. Furthermore, the frictionless, deformable and capacitive gas-liquid interface enables 3D manipulation of the fluid. More specifically, microflow regulation, consisting of resistive microchannels and a capacitive reservoir, exhibits the first-order fluidic response, analogous to the first-order $\boldsymbol{R} \boldsymbol{C}$ circuit. The flexible but geometrically confined interface can function as a pressure-limiting unit to restrict the maximal flow passing through. Furthermore, by connecting two first-order fluidic circuits with separate cutoff frequencies, flow ratecontrolled switching can be implemented using vertical expansion of the flexible interface. Both operations will be demonstrated and characterized in the following section as unique fluidic functions enabled by surface microfluidic networks.

\section{EXPERIMENTAL METHODS}

All planar microfluidic structures are fabricated by photopatterning a superhydrophobic nanocomposite as previously reported [9], in which polytetrafluoroethylene (PTFE) nanoparticle filler (Microdispers-200, Polyscience, Inc.) with extremely low chemical energy and nanotopology is embedded in a photopatternable SU-8 polymer matrix (SU-8 2050, MicroChem Corp.). The surface micromachining began with spin-coating a thick SU-8 photoresist layer (of $30 \mu \mathrm{m}$ thick) onto an acid-cleaned glass substrate. Subsequent to soft bake and selective UVexposure, the superhydrophobic nanocomposite material, dissolved in an ethanol solution, is spray-coated onto the exposed SU-8 layer as a uniform thin film (of $2 \mu \mathrm{m}$ thick). Followed by Isopropyl alcohol/DI water rinsing and blow drying, the substrate was immersed in SU-8 developer to form micropatterns with extreme wettability. To establish 3D microfluidic networks, two microfabricated planar fluidic substrates are placed face-to-face with a spacer and fluidic connected through miniature capillary bridges of the capacitive elements (reservoirs). In addition, the height of the spacer is adjusted according to different fluidic functions to be implemented.

Both DI water and aqueous-based color dyes (Waterman Paris) are used to conduct the fluidic evaluation. Inflow is directly injected to the inlet reservoir/channel from a computerprogrammable syringe pump (KD Scientific). The inlet pressure is determined by the fluidic curvature at the injection reservoir (i.e., hydraulic pressure). The outlet reservoir of a relatively large footprint is to provide zero pressure reference and insensitive to the 
volume change. The overall fluidic profile is screened through a horizontally placed digital stereomicroscope. The maximal flow rate is assessed at the steady-state condition for microflow regulation.

\section{RESULTS AND DISCUSSION}

To demonstrate the unique spatiotemporal (i.e., threedimensional and time-dependent) characteristics of surface microfluidics, two functional 3D microfluidic devices, a microflow regulator and a flow rate-controlled switch, have been fabricated and characterized. Using a capacitive fluidic element, the microflow regulator is able to limit the maximal flow through as well as to provide first-order fluidic damping. Whereas the flow rate controlled switch comprises two $\boldsymbol{R C}$ circuits in series, of which each stage has individual spatiotemporal response to different fluidic waveforms. Incorporating fluidic switches into 3D surface microfluidic networks, a microfluidic multiplexer has been devised with individual addressable microchannels using pulsed fluidic waveforms.

\section{Microflow Regulation}

Microflow regulators, providing dynamic control of microflow characteristics (e.g. flow rates and flow patterns), usually include active controlling unit or specific structural design, which involves multistep microfabrication and packaging [22-23]. In surface microfluidics, the spatiotemporal dependence of fluidic circuits enables a unique flow-limiting mechanism, that is, the maximal driven pressure is geometrically dependent. A flow passing through a circular reservoir with a primary pressuredependent capacitance connected to a more resistive surface microchannel. The flow rate established by the pressure head in the reservoir can be determined by the radius of curvature of the accumulated fluid in the reservoir. According to Laplace equation, a smaller radius of curvature leads to a higher pressure across the gas-liquid interface. Therefore, the maximal pressure head is achieved when the fluid forms a hemi-spherical dome shape (i.e., the radius of curvature equal to that of the reservoir).The growing hemi-spherical dome with decreasing pressure head would lead to positive feedback with mechanical instability, and eventually overflow to superhydrophobic surface.

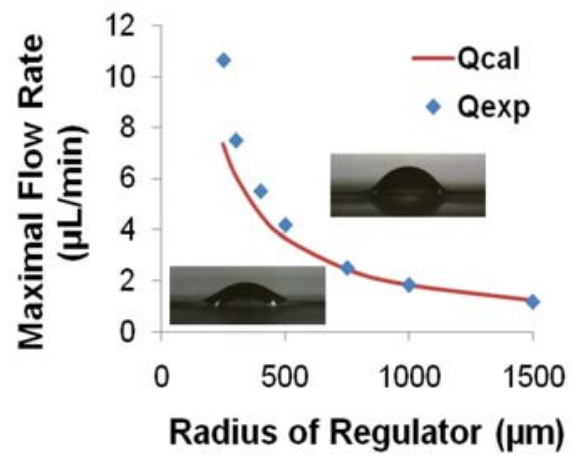

Figure 3: Experimental and computational results of the maximal flow rates in the flow-regulating reservoirs with different radii. The inserted snapshots indicate the steady state of the two regulators with $1 \mathrm{~mm}$ and $2 \mathrm{~mm}$ diameters' reservoirs, respectively.

To investigate the microflow regulation, the controlling reservoirs with different sizes from $500 \mu \mathrm{m}$ to $3 \mathrm{~mm}$ in diameter are connected to a straight microchannel with constant resistance (of $200 \mu \mathrm{m}$ in width, $40 \mu \mathrm{m}$ in depth, and $2 \mathrm{~cm}$ in length). Figure 3 shows the experimental measurements in comparison with the computational results of the maximal flow rates in the flow- regulating reservoirs with different radii. As can be seen, the results are well matched in the large reservoir configurations; however, a slight divergence from theoretical predictions has been observed in small reservoirs, where the stray capacitance and deformed interface of the microchannel contribute to additive fluidic conductance. The inserts in Figure 3 show the steady state liquid profile on circular reservoirs with diameters of $1 \mathrm{~mm}$ (bottom left) and $2 \mathrm{~mm}$ (top right). In 1mm-diameter one, the liquid height change in the channel which is comparable with that in the reservoir, should be considered when doing the calculations.

\section{Flow Rate-Controlled Switching}

Combining the flexible surface microfluidic network with capillary bridges, a flow rate-controlled switch is devised. As mentioned previously, the vertical expansion of gas-liquid interface of a capacitive element (reservoir) can be adjusted by the magnitude of inflow. By connecting two capacitive-resistive units in series, flow rate-dependent activation can be achieved. As shown in Figure 4, in two circular reservoirs with different diameters, the height of liquid dome can be dynamically controlled by the injection flow rate. That is at lower injection rate, fluid goes directly through the first reservoir (closer to the injection end) towards the second one (the far end) and accumulate there; while injecting at higher rate, most of the liquid builds up inside the first reservoir due to the aforementioned flow regulation. As a result, it is capable of addressing individual capillary bridge contact between the bottom and top substrates using different pulsed fluidic waveform, which serves as flow-activated switch function in the open fluidic circuits. Figure 4 illustrates individual activation of capillary bridge contacts based on two capacitive elements (reservoir) with different radii by controlling pulsed-flow rate. The pulsed flow can selectively control fluidic accumulation and thereby connect the capillary bridge between two fluidic circuits of the top and bottom substrates. Once the liquid bridge is formed, the flow rate can be reduced to a much lower level to maintain the continuous fluidic connection, from which the pulsed flow can be further used to control the next stage of fluidic function.

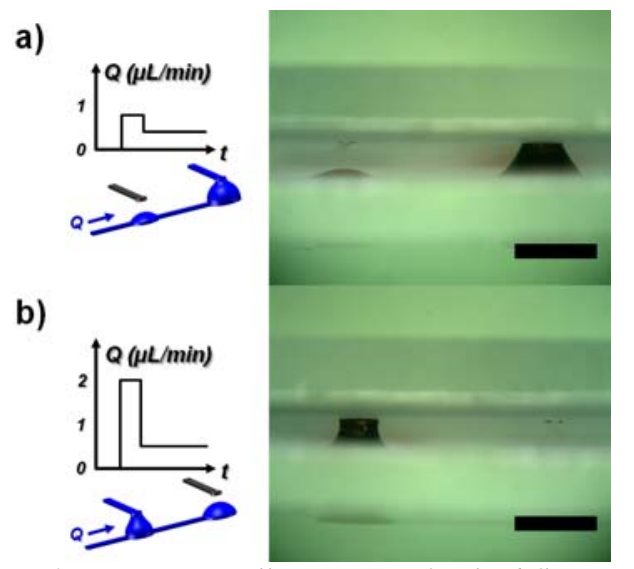

Figure 4: Microscopic illustrations of pulsed-flow switching along with applied pulsed flow waveforms (scale bars: $1 \mathrm{~mm}$ ).

Furthermore, based on the same principle demonstrated in pulsed-flow switch, a microfluidic multiplexing function can be implemented. Figure 5 shows the microfluidic multiplexer built on a 3D surface fluidic network. Under corresponding excitation flow pulses, the selected microchannel is individually activated. The multiplexer network can be easily extended to multistage configuration, while the simple pulse activation illustrates its potential in digital microfluidics. More sophisticated 3D surface fluidic operations are currently under investigation. 


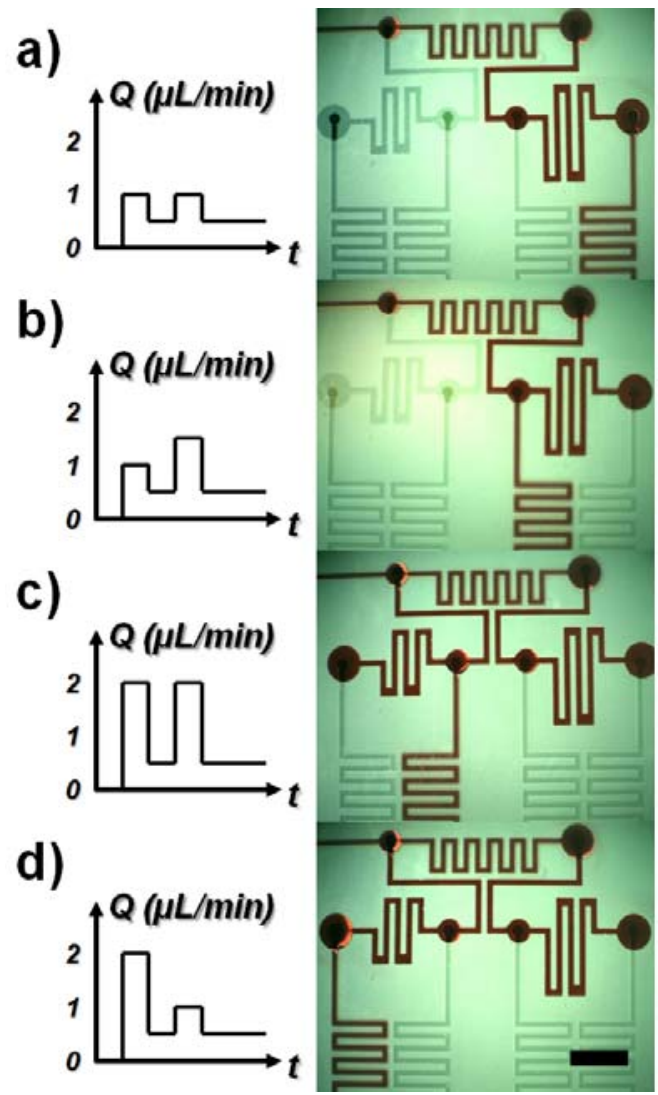

Figure 5: Demonstration of two-stage flow switching in a 3D microfluidic network with different switching-flow pulses (scale bar: $2 \mathrm{~mm}$ ).

\section{CONCLUSION}

By introducing a novel flexible frictionless gas-liquid interface, the surface microfluidic networks enable novel spatiotemporal fluidic manipulations on the planar surfaces, micropatterned by the nanocomposite material with extreme wettability. Detailed theoretical analysis, using the lumpedelement model, establishes both basic capacitive (circular reservoir) and resistive (straight microchannel) elements in the open fluidic platform. Further employing the deformable interface of a capacitive element, unique surface microfluidic functions have been implemented, i.e., microflow regulation and flow-rate controlled switching. Microflow regulator provides the functions of maximal flow restriction and fluctuation elimination, while the flow-rate controlled switching allows spatial activation of a specific path according the fluidic waveform. Furthermore, microfluidic multiplexers integrated with three-level flowswitching have been successfully devised, in which fluidic pulse signals can digitally activate individual microchannels. In summary, surface microfluidics, governed by distinct gas-liquid and solid-liquid interfaces, opens new possibilities to maneuver microflow in three-dimensional and time-dependent fashions, which can be further explored in digital and biomedical microfluidic applications.

\section{ACKNOWLEDGEMENT}

This work is in part supported by the National Science Foundation (Award Numbers: ECCS-0846502 and CMMI0944353), University of California, Davis, and China Scholarship Council (CSC). I would like to thank the Transducer Research
Foundation and National Institute of Standards and Technology, Boulder for the travel support to the conference.

\section{REFERENCES}

[1] D. J. Beebe et al., "Physics and applications of microfluidics in biology,” Annu Rev Biomed Eng, 4, 261, 2002.

[2] G. M. Whitesides, "The origins and the future of microfluidics," Nature, 442, 368, 2006.

[3] D. Therriault et al., "Chaotic mixing in three-dimensional microvascular networks fabricated by direct-write assembly,” Nat Mater, 2, 265, 2003.

[4] M. J. Swickrath et al., "Modulating passive micromixing in 2-D microfluidic devices via discontinuities in surface energy," Sensor Actuat B, 140, 656, 2009.

[5] W. Gu et al., "Computerized microfluidic cell culture using elastomeric channels and Braille displays,” Proc. Natl. Acad. Sci. USA, 101, 15861, 2004.

[6] G. M. Walker et al., "A passive pumping method for microfluidic devices,” Lab Chip, 2, 131, 2002.

[7] M. A. Unger, H. P. Chou, T. Thorsen et al., "Monolithic microfabricated valves and pumps by multilayer soft lithography," Science, 288, 113, 2000.

[8] B. D. Piorek et al., "Free-surface microfluidic control of surface-enhanced Raman spectroscopy for the optimized detection of airborne molecules," Proc. Natl. Acad. Sci. USA, 104, 18898, 2007.

[9] L. Hong, and T. Pan, "Photopatternable Superhydrophobic Nanocomposites for Microfabrication,” J. Microelectromech. Syst, 1, 2010.

[10] M. Zimmermann et al., "Capillary pumps for autonomous capillary systems,” Lab Chip, 7, 119, 2007.

[11] B. Zhao et al., "Principles of surface-directed liquid flow in microfluidic channels,” Anal. Chem., 74, 4259, 2002.

[12] S. Bouaidat et al., "Surface-directed capillary system; theory, experiments and applications," Lab Chip, 5, 827, 2005.

[13] J. West et al., "Microplasma writing for surface-directed millifluidics," Lab Chip, 7, 981, 2007.

[14] M. Watanabe, "Surface-directed channels filled with organic solvents,” Lab Chip, vol. 9, no. 8, pp. 1143, 2009.

[15] B. Zhao et al., "Surafce-directed liquid flow inside microchannels," Science, 291, 4, 2001.

[16] P. Lam et al., "Surface-tension-confined microfluidics," Langmuir, 18, 948, 2002.

[17] J. S. H. Lee et al., "Electrokinetic flow in a free surfaceguided microchannel," J Appl Phys, 99, 2006.

[18] M. Watanabe, "Microfluidic devices easily created using an office inkjet printer," Microfluid Nanofluid, 8, 403, 2010.

[19] S. Y. Yang et al., "Integration of a surface-directed microfluidic system with an organic electrochemical transistor array for multi-analyte biosensors,” Lab Chip, 9, 704, 2009.

[20] H. Gau et al., "Liquid morphologies on structured surfaces: From microchannels to microchips,” Science, 283, 46, 1999.

[21] L. Hong, and T. Pan, "Surface microfluidics fabricated by superhydrophobic nanocomposite photoresist," in MEMS 2010, Hongkong, 2010.

[22] B. Z. Yang et al., "A planar compliance-based self-adaptive microfluid variable resistor,” J Microelectromech S, 16, 411, 2007.

[23] W. Xu et al., “A vapor based microfluidic flow regulator," Sensor Actuat B, 142, 355, 2009.

\section{CONTACT}

*Tingrui Pan, tel: +1-530-754-9508; tingrui@ucdavis.edu 